\title{
The Prevalence and Prognosis of Resistant Hypertension in Patients with Heart Failure
}

\author{
Chun-Na Jin, Ming Liu, Jing-Ping Sun, Fang Fang, Yong-Na Wen, Cheuk-Man Yu, \\ Alex Pui-Wai Lee*
}

Division of Cardiology, Li Ka Shing Institute of Health Sciences, Department of Medicine and Therapeutics, Prince of Wales Hospital, The Chinese University of Hong Kong, Hong Kong SAR, People's Republic of China

*alexpwlee@cuhk.edu.hk

\section{Abstract}

Background: Resistant hypertension is associated with adverse clinical outcome in hypertensive patients. However, the prognostic significance of resistant hypertension in patients with heart failure remains uncertain.

Methods and Results: The 1 year survival and heart failure re-hospitalization rate of 1288 consecutive patients admitted to a university hospital for either newly diagnosed heart failure or an exacerbation of prior chronic heart failure was analyzed. Resistant hypertension was defined as uncontrolled blood pressure $(>140 / 90 \mathrm{mmHg}$ ) despite being compliant with an antihypertensive regimen that

\section{OPEN ACCESS}

Citation: Jin C-N, Liu M, Sun J-P, Fang F, Wen YN, et al. (2014) The Prevalence and Prognosis of Resistant Hypertension in Patients with Heart Failure. PLoS ONE 9(12): e114958. doi:10.1371/ journal.pone.0114958

Editor: Masaru Katoh, National Cancer Center, Japan

Received: August 29, 2014

Accepted: November 17, 2014

Published: December 9, 2014

Copyright: (c) 2014 Jin et al. This is an openaccess article distributed under the terms of the Creative Commons Attribution License, which permits unrestricted use, distribution, and reproduction in any medium, provided the original author and source are credited.

Data Availability: The authors confirm that all data underlying the findings are fully available without restriction. All relevant data are within the paper.

Funding: The authors received no specific funding for this work.

Competing Interests: The authors have declared that no competing interests exist. includes 3 or more drugs (including a diuretic). A total of $176(13.7 \%)$ heart failure patients had resistant hypertension. There was no difference in all cause mortality, cardiovascular mortality, and heart failure related re-hospitalization between patients with versus without resistant hypertension. Diabetes [hazard ratio $=1.62$, $95 \%$ confidence interval $=1.13-2.34 ; P=0.010]$ and serum sodium $>139 \mathrm{mmol} / \mathrm{L}$ (hazard ratio $=1.54,95 \%$ confidence interval $=1.06-2.23 ; P=0.024$ ) were independently associated with resistant hypertension. Patients with resistant hypertension had a relatively higher survival rate $(86.9 \%$ vs. $83.8 \%)$, although the difference was not significant (log-rank $\left.x^{2}=1.00, P=0.317\right)$. In patients with reduced ejection fraction, heart failure related re-hospitalization was significantly lower in patients with resistant hypertension ( $45.8 \%$ vs. $59.1 \%, P=0.050)$. Conclusions: Resistant hypertension appears to be not associated with adverse clinical outcome in patients with heart failure, in fact may be a protective factor for reduced heart failure related re-hospitalization in patients with reduced ejection fraction. 


\section{Introduction}

Hypertension is a major public health problem with a global prevalence ranging from about $20 \%$ to $40 \%$ [1-2] . Resistant hypertension, defined by the USA Joint National Committee (JNC)-7 as failure to achieve goal blood pressure (BP) $(>140 / 90 \mathrm{mmHg}$ for the overall population and $>130 / 80 \mathrm{mmHg}$ for those with diabetes or chronic kidney disease) despite adhering to maximum tolerated doses of three antihypertensive drugs including a diuretics, is associated with a higher risk of cardiovascular morbidity and mortality [3] . A similar definition was adopted by the American Heart Association and the European Society of Cardiology.

Despite a standardized definition, the actual prevalence of resistant hypertension in the general population is difficult to estimate. According to the previous population-based studies [ $\underline{4}-\underline{5}]$, retrospective studies [ $[\underline{-}-\underline{7}]$ and outcome trials [ $\underline{8}-$ 9], resistant hypertension is not uncommon in hypertensive population and the estimated prevalence of resistant hypertension varies from study to study. Moreover, resistant hypertension was associated with a significantly increased risk of adverse cardiovascular events compared with non-resistant hypertension and represents an important public health issue. Heart failure is another ubiquitous cause of mortality and morbidity. There is a significant overlap between patients with heart failure and hypertension. In Enhanced Feedback for Effective Cardiac Treatment (EFFECT) study, where 69\% patients with reduced ejection fraction (HFREF) and 31\% patients with preserved ejection fraction (HFPEF), nearly 51\% of total heart failure patients had evidence of hypertension [10]. The Systolic Hypertension in Elderly Program (SHEP) trial included 4736 people $>60$ years of age and demonstrated that reducing blood pressure from $170 / 77$ to $143 / 78 \mathrm{~mm}$ $\mathrm{Hg}$ reduced heart failure events by $48 \%$ [11]. Up to now, there is little data in regard to the prevalence of resistant hypertension in HF patients. When heart failure co-existent with resistant hypertension, the combination is likely to be associated with deleterious consequence.

Accordingly, this study will firstly focus on investigating the prevalence of resistant hypertension and the optimal $\mathrm{BP}$ control rate in our heart failure patients both with reduced and preserved ejection fraction (EF). Clinical outcomes, such as 1-year all-cause mortality, cardiovascular mortality and heart failure related rehospitalization, also will be well assessed in heart failure patients with or without resistant hypertension.

\section{Methods}

\subsection{Patients population}

Consecutive patients presented to a tertiary teaching hospital with either newly diagnosed heart failure or an exacerbation of prior chronic HF were prospectively studied. The diagnosis of heart failure was established according to the clinical Framingham criteria [12]. Patients younger than 18 year-old or refusing to 
participating in this study were excluded. The study was planned according to the Declaration of Helsinki and approved by Joint Chinese University of Hong Kong New Territories East Cluster Clinical Research Ethics Committee and all patients provided written informed consent to participate in this study.

\subsection{Baseline measurements}

Demography characteristics and clinical data, including the medical history, medications, cardiovascular risk factors, and associate co-morbidities, were collected using a standardized case report form that was completed at the every study visit. Complementary data collection included electrocardiography, echocardiography, and laboratory tests during the follow up visits. Discharge prescription of the main cardiovascular therapeutics classes was recorded.

Baseline BP was measured in seated position after at least 5 minutes of rest. BP measurement in atrial fibrillation, particularly when the ventricular rhythm is highly irregular, will at best constitute a rough estimate, the validity of which can perhaps be improved upon only by using repeated measurements. The treatment goal of hypertension is to maintain the systolic BP (SBP) and diastolic BP (DBP) below $140 \mathrm{mmHg}$ and $90 \mathrm{~mm} \mathrm{Hg}$, respectively. Antihypertensive medications were grouped based on drug class, mainly including diuretics, angiotensinconverting enzyme inhibitors (ACEIs)/angiotensin II receptors blockers (ARBs), calcium channel blockers (CCBs), beta-blockers and aldosterone receptor antagonists(ARAs). Resistant hypertension was diagnosed as uncontrolled BP despite using of more than 3 antihypertensive medications, including a diuretics.

Comprehensive two-dimensional Doppler transthoracic echocardiography was performed in all participants (Vivid Five or Seven, General Electric, Milwaukee, WI, USA) using a $2.5 \mathrm{MHz}$ probe. All images were digitally stored with at least three cardiac cycles for off-line analysis. The left ventricular (LV) volumes and left ventricular ejection fraction (LVEF) were assessed by the biplane Simpson's method. At least three consecutive beats in sinus rhythm were measured and averaged. Patients with an EF of $<50 \%$ were classified as HFREF, and those with an EF of greater than or equal to $50 \%$ were classified as HFPEF.

\subsection{Ascertainment of outcomes}

The primary outcome for the study was all-cause mortality within 1-year followup. Secondary outcomes included cardiovascular death and heart failure related re-hospitalization within 1-year follow-up. Outcomes were not adjudicated, but based on physician reporting at the time of follow-up. Cardiovascular death included fatal stroke, fatal myocardial infarction, death attributed to congestive heart failure, sudden cardiac death, pulmonary embolism and other cardiac death.

\subsection{Statistical analysis}

Continuous variables are expressed as the mean $\pm \mathrm{SD}$, categorical data are presented as the absolute numbers and percentages. Differences between groups 
were evaluated using the Student t-test for continuous data, the chi-square test for binomial or nominal variables (or Fisher's exact test, if appropriate). Logistic regression was conducted to screen factors at baseline associated with resistant hypertension. Hazard ratios (HR) and their 95\% confidence interval (CI) were calculated. Kaplan-Meier survival curves were constructed to demonstrate 1-year survival in patients with or without resistant hypertension. The log-rank test was used to determine if actuarial survival was significantly different. Statistical significance was considered as a 2-tailed probability of $<0.05$. Statistical analysis was performed using SPSS software, version 20.0 for Windows (SPSS, Inc., Chicago, IL, USA).

\section{Results}

\subsection{Baseline characteristics and follow-up endpoints of the sample} 1288 patients with heart failure enrolled in this registry (May 2006 to December 2010) were entered into the final analysis. Among all the patients included, mean age was 75.2 \pm 11.9 years, ranged from 31-102 years; $713(55.4 \%)$ patients were female; 519 (40.3\%) were HFREF patients; 381 (29.6\%) patients received more than 3 antihypertensive medications; 679 (52.7\%) patients reached optimal BP target; a total of $176(13.7 \%)$ heart failure patients were found to combine with resistant hypertension. All patients were followed up for 1 year, 203 (15.8\%) patients died within 1-year follow-up, among them 45.3\% (92) patients were belong to cardiovascular death; $657(51.0 \%)$ patients had at least 1 episode of heart failure related re-hospitalization.

\subsection{Differential characteristics and the prognosis of patient groups defined by with or without resistant hypertension}

Among all the heart failure patients with resistant hypertension, the 5 most used classes of antihypertensive agents included diuretics (100\%), beta-blockers (86.4\%), ACEIs/ARBs (84.1\%), CCBs (31.2\%), and ARAs (13.1\%). The baseline characteristics for patients with or without resistant hypertension were shown in Table 1. Patients with resistant hypertension had a higher incidence of diabetes, a remarkably higher level of serum sodium and albumin, and were more commonly prescribed with statins. Logistic-regression analysis further revealed that patients with higher serum sodium $(>139 \mathrm{mmol} / \mathrm{L})(\mathrm{HR}=1.54,95 \% \mathrm{CI}=1.06-2.23$; $P=0.024)$ and with a history of diabetes $(\mathrm{HR}=1.62,95 \% \mathrm{CI}=1.13-2.34 ; P=0.010)$ at baseline were more likely to be resistant hypertension (Table 2).

Kaplan-Meier analysis showed that patients with resistant hypertension had a relatively higher survival rate $(86.9 \%$ vs. $83.8 \%)$, while the difference was not significant (log-rank $\left.x^{2}=1.00 ; P=0.317\right)$ at 1 -year follow-up. There were no difference in heart failure related re-hospitalization $(51.4 \%$ vs. $48.3 \% ; P=0.438)$ and cardiovascular mortality $(43.9 \%$ vs. $56.5 \%$; $P=0.252)$ between the two groups during a follow-up of 1 year (Table 3 ). 
Table 1. Baseline characteristics for heart failure patients with or without resistant hypertension.

\begin{tabular}{|c|c|c|c|}
\hline Parameters & $\begin{array}{l}\text { Non-RHTN } \\
(n=1112)\end{array}$ & $\begin{array}{l}\text { RHTN } \\
(n=176)\end{array}$ & $P$ \\
\hline Age, years & $75.3 \pm 11.9$ & $74.4 \pm 11.8$ & 0.338 \\
\hline Female, no. (\%) & $612(55.0)$ & $101(57.4)$ & 0.560 \\
\hline NYHA III-IV, no. (\%) & $799(77.7)$ & $131(80.4)$ & 0.448 \\
\hline Smokers, no. (\%) & $348(32.1)$ & $48(28.7)$ & 0.381 \\
\hline Heart rate, bpm & $90 \pm 24$ & $91 \pm 24$ & 0.862 \\
\hline LVEF, \% & $51 \pm 16$ & $52 \pm 15$ & 0.308 \\
\hline HF with preserved EF, no. (\%) & $642(58.4)$ & $112(65.1)$ & 0.096 \\
\hline $\mathrm{SBP}, \mathrm{mmHg}$ & $134 \pm 25$ & $157 \pm 18$ & $<0.001$ \\
\hline $\mathrm{DBP}, \mathrm{mmHg}$ & $72 \pm 14$ & $81 \pm 15$ & $<0.001$ \\
\hline \multicolumn{4}{|l|}{ Laboratory Test } \\
\hline Sodium, mmol/L & $138.9 \pm 4.5$ & $139.8 \pm 3.6$ & 0.007 \\
\hline Potassium, $\mathrm{mmol} / \mathrm{L}$ & $4.07 \pm 0.62$ & $4.05 \pm 0.55$ & 0.602 \\
\hline Creatinine, umol/L & $138.5 \pm 96.7$ & $145.0 \pm 116.7$ & 0.424 \\
\hline Blood urea nitrogen, $\mathrm{mmol} / \mathrm{L}$ & $10.1 \pm 6.2$ & $9.8 \pm 5.8$ & 0.551 \\
\hline Albumin (>34 g/L), no. (\%) & $827(76.6)$ & $137(83.0)$ & 0.067 \\
\hline \multicolumn{4}{|l|}{ Medical history, no. (\%) } \\
\hline New on-set HF & $644(57.9)$ & $102(58.0)$ & 0.992 \\
\hline Hypertension & $826(74.3)$ & $145(82.4)$ & 0.020 \\
\hline Diabetes & $414(37.3)$ & $93(52.8)$ & $<0.001$ \\
\hline Coronary heart disease & $341(29.6)$ & $54(30.7)$ & 0.779 \\
\hline Atrial fibrillation & $319(28.9)$ & $43(24.4)$ & 0.220 \\
\hline COPD/Asthma & $123(12.5)$ & $11(7.5)$ & 0.081 \\
\hline Stoke/TIA & $157(14.2)$ & $28(15.9)$ & 0.538 \\
\hline Hyperlipidemia & 194(17.6) & $35(20.1)$ & 0.413 \\
\hline Chronic Kidney Disease & $182(16.5)$ & $31(17.6)$ & 0.705 \\
\hline Anemia & $450(41.1)$ & $63(37.3)$ & 0.342 \\
\hline \multicolumn{4}{|l|}{ Medication, no. (\%) } \\
\hline Regular nitrates & $252(22.8)$ & $42(24.4)$ & 0.645 \\
\hline Digoxin & $164(14.8)$ & $31(18.0)$ & 0.278 \\
\hline Aspirin & $596(53.6)$ & $99(56.6)$ & 0.470 \\
\hline Wafarin & $181(16.4)$ & $30(17.4)$ & 0.727 \\
\hline Statins & $369(33.2)$ & $73(41.7)$ & 0.028 \\
\hline
\end{tabular}

NYHA indicates New York Heart Association; LVEF, left ventricular ejection fraction; HF, heart failure; SBP, systolic blood pressure; DBP, diastolic blood pressure; RHTN, resistant hypertension; COPD, chronic obstructive pulmonary disease; and TIA, transient ischemic attack.

doi:10.1371/journal.pone.0114958.t001

\subsection{Differential characteristics and the prognosis of patient groups defined by HEREF or HFPEF}

In patients with HFREF, the prevalence of resistant hypertension was significantly higher $(15.2 \%$ vs. $11.4 \%, P=0.049)$. Within 1-year follow-up, all-cause mortality was $19.7 \%$ in patients with HFREF, significantly higher than patients with HFPEF

(19.7 vs. $13.1 \%, P=0.002$ ); heart failure related re-hospitalization was also higher 
Table 2. Logistic-regression of resistant hypertension for heart failure patients.

\begin{tabular}{|c|c|c|c|c|c|c|}
\hline \multirow[t]{2}{*}{ Variable } & \multicolumn{3}{|c|}{ Univariate analysis } & \multicolumn{3}{|c|}{ Multivariate analysis } \\
\hline & HR & $95 \% \mathrm{Cl}$ & $P$ & 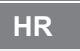 & $95 \% \mathrm{Cl}$ & $P$ \\
\hline Albumin(>34 g/L) & 1.49 & $0.97-2.29$ & 0.069 & - & - & - \\
\hline Sodium(>139.5 mmol/L) & 1.50 & $1.08-2.09$ & 0.016 & 1.54 & $1.06-2.23$ & 0.024 \\
\hline Statins used & 1.44 & $1.04-1.99$ & 0.029 & - & - & - \\
\hline Diabetes & 1.88 & $1.37-2.59$ & $<0.001$ & 1.62 & $1.13-2.34$ & 0.010 \\
\hline COPD/Asthma & 0.57 & $0.30-1.08$ & 0.085 & - & - & - \\
\hline
\end{tabular}

$\mathrm{HR}$ indicates hazard ratio; $\mathrm{Cl}$, confidence interval; and COPD, chronic obstructive pulmonary disease.

doi:10.1371/journal.pone.0114958.t002

in patients with HFREF ( $57.6 \%$ vs. $46.6 \%$; $P<0.001$ ); cardiovascular mortality was similar in both subgroups $(49.0 \%$ vs. $41.6 \% ; P=0.287)$. When resistant hypertension was considered, all-cause mortality and cardiovascular mortality were similar in the two groups regardless of patient with reduced or preserved EF. In HFREF group, heart failure related re-hospitalization was significantly higher in patients without resistant hypertension than those with resistant hypertension (59.1\% vs. $45.8 \% ; P=0.050$ ), while this difference was not found in HFPEF group (45.9\% vs. $50.0 \% ; P=0.415$ ) (Table 4 ).

\section{Discussion}

\subsection{The prevalence of resistant hypertension in patients with heart failure}

We totally studied 1288 patients with heart failure in the final analysis. The percentage of resistant hypertension in heart failure patients was $13.7 \%$.

Compared to patients with HFREF, the prevalence of resistant hypertension was significantly higher in patients with HFPEF. The optimal BP control rate was only $52.7 \%$ among all the patients. Patients with a history of diabetes and higher serum sodium $(>139 \mathrm{mmol} / \mathrm{L})$ at baseline were more likely to combine with resistant hypertension, which had been well demonstrated as risk factors contributing to resistant hypertension in general population previously [3] .

Current prevalence estimated for resistant hypertension in other population subgroups vary. Population-based studies, such as National Health and Nutrition

Table 3. 1-year outcomes comparison for heart failure patients with or without resistant hypertension.

\begin{tabular}{l|l|l|l|}
\hline Outcomes, no. (\%) & Heart failure patients & $P$ \\
\hline & Non-RHTN $(\mathbf{n}=1112)$ & RHTN $(\mathbf{n = 1 7 8 )}$ & \\
\hline All-cause mortality & $180(16.2)$ & $23(13.1)$ & 0.291 \\
Cardiovascular mortality & $79(43.9)$ & $13(56.5)$ & 0.252 \\
HF Re-hospitalization & $572(51.4)$ & $85(48.3)$ & 0.438 \\
\hline
\end{tabular}

HF indicates heart failure; and RHTN, resistant hypertension.

doi:10.1371/journal.pone.0114958.t003 
Table 4. 1-year outcomes comparison for subgroup analysis based on ejection fraction in patients with or without resistant hypertension.

\begin{tabular}{|c|c|c|c|c|c|c|}
\hline \multirow[t]{2}{*}{ Outcomes, no. (\%) } & \multicolumn{3}{|c|}{ HFREF patients $(n=519)$} & \multicolumn{3}{|c|}{ HFPEF patients $(n=769)$} \\
\hline & Non-RHTN $(n=460)$ & RHTN $(n=59)$ & $P$ & Non-RHTN $(n=652)$ & RHTN (n=117) & $P$ \\
\hline All-cause mortality & $93(20.2)$ & $9(15.3)$ & 0.366 & $87(13.3)$ & $14(12.0)$ & 0.685 \\
\hline Cardiovascular mortality & $46(49.5)$ & $4(44.4)$ & 0.774 & $33(37.9)$ & $9(64.3)$ & 0.063 \\
\hline HF Re-hospitalization & $272(59.1)$ & $27(45.8)$ & 0.050 & $300(46.0)$ & $58(49.6)$ & 0.477 \\
\hline
\end{tabular}

HFREF indicates heart failure with reduced ejection fraction; HFPEF, heart failure with preserved ejection fraction; and RHTN, resistant hypertension.

doi:10.1371/journal.pone.0114958.t004

Examination Survey (NHANES), reported that the prevalence of resistant hypertension was $8-12 \%$ among adult hypertensive patients (6-9 million people) [13]. Several recent studies estimated the prevalence of resistant hypertension was between $12.8 \%$ and $28 \%$ among hypertensive patients receiving anti-hypertension treatment $[\underline{4}-\underline{5}, \underline{14}]$. Hitherto, the prevalence of resistant hypertension has not been well defined in heart failure patients. A greater understanding of the prevalence of resistant hypertension is important to improve the management of these patients. Swedish Heart Failure Registry (S-HFR) had shown that the percentage of heart failure patients with SBP more than $140 \mathrm{~mm} \mathrm{Hg}$ was $22.4 \%$ [15], suggesting that heart failure patients without optimal BP control is common. Our current study firstly reported the overall prevalence of resistant hypertension was $13.7 \%$ in heart failure patients, which felled in the range of reported prevalence in hypertensive patients $[\underline{4}-\underline{5}, \underline{14}]$, however this study was not primarily designed as a survey to detect true prevalence rates.

\subsection{The prognosis of heart failure patients with resistant hypertension within 1-year follow-up}

In the current study, heart failure patients with resistant hypertension didn't show a remarkably difference in the outcomes compared with those without resistant hypertension within 1 year follow up, suggesting that resistant hypertension appears not to be associated with increased 1 year mortality in heart failure patients. In contrast, precious studies had reported that resistant hypertension was associated with a significantly increased risk of adverse cardiovascular events in the rest of cardiac population [6]. Patients present with a long-standing history of poorly controlled hypertension had an unfavorable prognosis. When considering the different study population between ours and others, it is difficult to establish the exact role resistant hypertension had taken in heart failure patients.

Our study had shown the status of resistant hypertension, in another word, elevation of blood pressure above normal in heart failure patients, appears not to be associated with increased 1-year mortality in heart failure patients. As far as we are aware, the result we found was in keeping with the previous studies on heart failure patients. On one hand, a recent meta-analysis quantifying the paradoxical effect of higher SBP on mortality in chronic heart failure (mainly including HFREF) disclosed that the decrease in mortality rates associated with a $10 \mathrm{~mm} \mathrm{Hg}$ 
higher SBP was $13.0 \%$ (95\% CI: $10.6 \%$ to $15.4 \%$ ) in the heart failure population [16]. On the other hand, in several clinical trials involving patients with chronic heart failure [17] or acute heart failure [18-19], lower BPs were generally recognized to be an adverse prognostic marker in risk assessment of heart failure, which also were confirmed in both HFREF [20] and HFPEF [21]. Up to now, there was no clear cutoff value to define the optimal SBP control goal in heart failure patients. Data from the African-American Heart Failure Trial (A-HeFT) indicated best outcomes for the group with baseline SBP 126-140 mm Hg, whereas SBP below the median induced a small but significant increase in mortality, regardless of treatment assignment [22].

Patients with HFREF and HFPEF could potentially be viewed as 2 unique populations. Despite our incomplete understanding of the pathophysiologic progression leading to the development into systolic dysfunction or diastolic dysfunction, the benefits of treating hypertension are clear, and the dramatic improvement in outcomes had been seen when hypertension was controlled [23]. Current study disclosed that in patients with HFREF, all-cause mortality and heart failure related re-hospitalization were significantly higher than patients with HFPEF within 1-year follow-up. Large attention on heart failure patients with or without resistant hypertension had been taken in this study. Within 1-year followup, HF related re-hospitalization was lower in HFREF patients with resistant hypertension, which imply that resistant hypertension may not be a factor for increasing re-hospitalization in heart failure patients with reduced EF. Our study further found effects of resistant hypertension on 1-year mortality don't vary from HFPEF patients to HFREF patients, indicating that resistant hypertension appears not associated with adverse long-term clinical mortality.

\subsection{Clinical significance}

Current study has shown $13.7 \%$ heart failure patients accompanying with drugrelated resistant hypertension. Compared with heart failure patients without resistant hypertension, those with resistant hypertension received more aggressive antihypertensive treatment but similar outcomes. Resistant hypertension has the similar pathophysiologic mechanism to heart failure, as the excessive activation of renin-angiotensin-aldosterone system and sympathetic nervous system. It was known that heart failure patients with preserved EF benefited limitedly from the current available treatments, such as pharmacotherapy and cardiac resynchronization therapy. Over the past few years, there has been significant interest in device-based therapies, which modify sympathetic nerves activity for the management of patients with resistant hypertension, including renal denervation via radiofrequency catheter ablation of renal sympathetic nerves (RDT) [24] and baroreceptor activation therapy (BAT) [25-26]. There may be a theoretical concern in practice that patients with heart failure typically have normal or low $\mathrm{BP}$ and may be relying upon contributions from their sympathetic nerves activity to sustain BP and organ perfusion, while RDT and BAT therapy might further lower the BP. So, heart failure patients with resistant hypertension may be the 
optimal indication for these novel device-bases therapies. If confirmed effective, about one in sixth heart failure patients will benefit from these new therapies in our study.

\subsection{Limitations}

Certain limitations should be considered in the interpretation of the study results. Firstly, the present study used office-based BP measurements alone; ambulatory BP measurements may provide more accurate estimates of resistant hypertension and have been shown to be more prognostic [4] . However, office-based BP measurements reflect current practice and are used routinely in the management of heart failure patients. Another limitation is lack of information on medication dosing since optimal dosing constitutes an integral part of the definition of resistant hypertension, whereas some earlier studies have used a definition identical to ours $[\underline{4-5}, \underline{14}, 27]$. Maximally tolerated/adequate doses was referred by guidelines, but vary from person to person [3]. Besides that, BP is intrinsically related to the heart's ability to pump and decreases as a result of reduced cardiac output due to heart failure deterioration, which will underestimate the true percentage of resistant hypertension in heart failure patients. Furthermore, the sample size in our study is relatively small and the power to discriminate the difference of 1 year outcomes between patients with or without resistant hypertension is low. The present study population was drawn from a single center, so our finding may not be generalizable to other healthcare system. Finally, another limitation in our study is absence of the adjustment of the presence of moderate and severe diastolic dysfunction and left ventricular hypertrophy (LVH), which have been demonstrated as independently predictors of adverse clinical outcomes in patients with heart failure in the CHARM study [28-29]. However, most of the previous studies with the purpose of investigating the outcomes in patients with heart failure did not adjust these two parameters [10,30-41]. Only few studies adjusted them separately, but no positive association was found $[\underline{42-44}]$.

\section{Conclusions}

As far as we know, this is the first study focusing on the prevalence and prognosis of resistant hypertension in heart failure patients. Resistant hypertension is a common finding and appears not to be associated with increased 1-year mortality and heart failure related re-hospitalization in heart failure patients. Further studies are necessary to determine the correct pharmacologic treatment, as well as the effectiveness of some novel device-bases therapies, such as RDT and BAT, for heart failure patients with resistant hypertension. 


\section{Author Contributions}

Conceived and designed the experiments: APWL. Performed the experiments: CNJ ML YNW. Analyzed the data: CNJ. Contributed reagents/materials/analysis tools: APWL CMY. Contributed to the writing of the manuscript: CNJ. Helped to critically revise the manuscript: JPS FF.

\section{References}

1. Wolf-Maier K, Cooper RS, Banegas JR, Giampaoli S, Hense HW, et al. (2003) Hypertension prevalence and blood pressure levels in 6 European countries, Canada, and the United States. JAMA 289: 2363-2369.

2. Writing Group M, Roger VL, Go AS, Lloyd-Jones DM, Benjamin EJ, et al. (2012) Heart Disease and Stroke Statistics-2012 Update: A Report From the American Heart Association. Circulation 125: e2e220.

3. Chobanian AV, Bakris GL, Black HR, Cushman WC, Green LA, et al. (2003) The Seventh Report of the Joint National Committee on Prevention, Detection, Evaluation, and Treatment of High Blood Pressure: the JNC 7 report. JAMA 289: 2560-2572.

4. De La Sierra A, Segura J, Banegas JR, Gorostidi M, De La Cruz JJ, et al. (2011) Clinical features of 8295 patients with resistant hypertension classified on the basis of ambulatory blood pressure monitoring. Hypertension 57: 898-902.

5. Egan BM, Zhao Y, Axon RN, Brzezinski WA, Ferdinand KC (2011) Uncontrolled and apparent treatment resistant hypertension in the United States, 1988 to 2008. Circulation 124: 1046-1058.

6. Daugherty SL, Powers JD, Magid DJ, Tavel HM, Masoudi FA, et al. (2012) Incidence and prognosis of resistant hypertension in hypertensive patients. Circulation 125: 1635-1642.

7. McAdam-Marx C, Ye X, Sung JC, Brixner DI, Kahler KH (2009) Results of a retrospective, observational pilot study using electronic medical records to assess the prevalence and characteristics of patients with resistant hypertension in an ambulatory care setting. Clin Ther 31: 1116-1123.

8. Gupta AK, Nasothimiou EG, Chang CL, Sever PS, Dahlof B, et al. (2011) Baseline predictors of resistant hypertension in the Anglo-Scandinavian Cardiac Outcome Trial (ASCOT): a risk score to identify those at high-risk. J Hypertens 29: 2004-2013.

9. Group AOaCftACR (2002) Major outcomes in high-risk hypertensive patients randomized to angiotensin-converting enzyme inhibitor or calcium channel blocker vs diuretic: The antihypertensive and lipid-lowering treatment to prevent heart attack trial (allhat). JAMA 288: 2981-2997.

10. Bhatia RS, Tu JV, Lee DS, Austin PC, Fang J, et al. (2006) Outcome of heart failure with preserved ejection fraction in a population-based study. N Engl J Med 355: 260-269.

11. Kostis JB, Davis BR, Cutler J, Grimm RH Jr, Berge KG, et al. (1997) Prevention of heart failure by antihypertensive drug treatment in older persons with isolated systolic hypertension. SHEP Cooperative Research Group. JAMA 278: 212-216.

12. McKee PA, Castelli WP, McNamara PM, Kannel WB (1971) The natural history of congestive heart failure: the Framingham study. N Engl J Med 285: 1441-1446.

13. Sarafidis PA, Georgianos P, Bakris GL (2013) Resistant hypertension - Its identification and epidemiology. Nat Rev Nephrol 9: 51-58.

14. Persell S (2011) Prevalence of resistant hypertension in the United States 2003-2008. Hypertension 57: 1076-1080.

15. Jonsson A, Edner M, Alehagen U, Dahlstrom U (2010) Heart failure registry: a valuable tool for improving the management of patients with heart failure. Eur J Heart Fail 12: 25-31.

16. Raphael CE, Whinnett ZI, Davies JE, Fontana M, Ferenczi EA, et al. (2009) Quantifying the paradoxical effect of higher systolic blood pressure on mortality in chronic heart failure. Heart 95: 56-62. 
17. Grigorian-Shamagian L, Gonzalez-JuAnatey JR, Vazquez R, Cinca J, Bayes-Genis A, et al. (2008) Association of blood pressure and its evolving changes with the survival of patients with heart failure. J Card Fail 14: 561-568.

18. Abraham WT, Fonarow GC, Albert NM, Stough WG, Gheorghiade M, et al. (2008) Predictors of inhospital mortality in patients hospitalized for heart failure: insights from the Organized Program to Initiate Lifesaving Treatment in Hospitalized Patients with Heart Failure (OPTIMIZE-HF). J Am Coll Cardiol 52: 347-356.

19. Nunez J, Nunez E, Fonarow GC, Sanchis J, Bodi V, et al. (2010) Differential prognostic effect of systolic blood pressure on mortality according to left-ventricular function in patients with acute heart failure. Eur J Heart Fail 12: 38-44.

20. Lee TT, Chen J, Cohen DJ, Tsao L (2006) The association between blood pressure and mortality in patients with heart failure. Am Heart J 151: 76-83.

21. Buiciuc O, Rusinaru D, Levy F, Peltier M, Slama M, et al. (2011) Low systolic blood pressure at admission predicts long-term mortality in heart failure with preserved ejection fraction. $\mathrm{J}$ Card Fail 17: 907-915.

22. Anand IS, Tam SW, Rector TS, Taylor AL, Sabolinski ML, et al. (2007) Influence of blood pressure on the effectiveness of a fixed-dose combination of isosorbide dinitrate and hydralazine in the AfricanAmerican Heart Failure Trial. J Am Coll Cardiol 49: 32-39.

23. Moser M, Hebert PR (1996) Prevention of disease progression, left ventricular hypertrophy and congestive heart failure in hypertension treatment trials. J Am Coll Cardiol 27: 1214-1218.

24. Davies JE, Manisty CH, Petraco R, Barron AJ, Unsworth B, et al. (2013) First-in-man safety evaluation of renal denervation for chronic systolic heart failure: Primary outcome from REACH-Pilot study. Int J Cardiol 162: 189-192.

25. Bisognano JD, De Leeuw P, Bach DS, Lovett EG, Kaufman CL (2009) Improved functional capacity and cardiovascular structure after baroreflex activation therapy(trademark) in resistant hypertension patients with symptomatic heart failure: Results from european and united states trials of the Rheos(registered trademark) system. J Card Fail 15: S63.

26. Fagard RH, Celis H, Thijs L, Wouters S (2009) Regression of left ventricular mass by antihypertensive treatment: a meta-analysis of randomized comparative studies. Hypertension 54: 1084-1091.

27. Sarafidis PA, Bakris GL (2008) Resistant Hypertension. An Overview of Evaluation and Treatment. J Am Coll Cardiol 52: 1749-1757.

28. Persson H, Lonn E, Edner M, Baruch L, Lang CC, et al. (2007) Diastolic dysfunction in heart failure with preserved systolic function: need for objective evidence:results from the CHARM Echocardiographic Substudy-CHARMES. J Am Coll Cardiol 49: 687-694.

29. Hawkins NM, Wang D, McMurray JJV, Pfeffer MA, Swedberg K, et al. (2007) Prevalence and prognostic implications of electrocardiographic left ventricular hypertrophy in heart failure: evidence from the CHARM programme. Heart 93: 59-64.

30. De Blois J, Simard S, Atar D, Agewall S (2010) COPD predicts mortality in HF: the Norwegian Heart Failure Registry. J Card Fail 16: 225-229.

31. Harjola VP, Follath F, Nieminen MS, Brutsaert D, Dickstein K, et al. (2010) Characteristics, outcomes, and predictors of mortality at 3 months and 1 year in patients hospitalized for acute heart failure. Eur J Heart Fail 12: 239-248.

32. Goldberg RJ, Ciampa J, Lessard D, Meyer TE, Spencer FA (2007) Long-term survival after heart failure: a contemporary population-based perspective. Arch Intern Med 167: 490-496.

33. Pulignano G, Del Sindaco D, Tavazzi L, Lucci D, Gorini M, et al. (2002) Clinical features and outcomes of elderly outpatients with heart failure followed up in hospital cardiology units: data from a large nationwide cardiology database (IN-CHF Registry). Am Heart J 143: 45-55.

34. Fonarow GC, Abraham WT, Albert NM, Stough WG, Gheorghiade M, et al. (2008) Factors identified as precipitating hospital admissions for heart failure and clinical outcomes: findings from OPTIMIZE-HF. Arch Intern Med 168: 847-854

35. Lee DS, Austin PC, Rouleau JL, Liu PP, Naimark D, et al. (2003) Predicting mortality among patients hospitalized for heart failure: derivation and validation of a clinical model. JAMA 290: 2581-2587. 
36. Anker SD, Ponikowski P, Varney S, Chua TP, Clark AL, et al. (1997) Wasting as independent risk factor for mortality in chronic heart failure. Lancet 349: 1050-1053.

37. Badheka AO, Rathod A, Kizilbash MA, Bhardwaj A, Ali O, et al. (2011) Comparison of mortality and morbidity in patients with atrial fibrillation and heart failure with preserved versus decreased left ventricular ejection fraction. Am J Cardiol 108: 1283-1288.

38. Brophy JM, Dagenais GR, McSherry F, Williford W, Yusuf S (2004) A multivariate model for predicting mortality in patients with heart failure and systolic dysfunction. Am J Med 116: 300-304.

39. Cygankiewicz I, Zareba W, Vazquez R, Bayes-Genis A, Pascual D, et al. (2009) Risk stratification of mortality in patients with heart failure and left ventricular ejection fraction $>35 \%$. Am J Cardiol 103 1003-1010.

40. Horwich TB, Kalantar-Zadeh K, MacLellan RW, Fonarow GC (2008) Albumin levels predict survival in patients with systolic heart failure. Am Heart J 155: 883-889.

41. Liu M, Chan CP, Yan BP, Zhang Q, Lam YY, et al. (2012) Albumin levels predict survival in patients with heart failure and preserved ejection fraction. Eur J Heart Fail 14: 39-44.

42. Pocock SJ, Wang D, Pfeffer MA, Yusuf S, McMurray JJ, et al. (2006) Predictors of mortality and morbidity in patients with chronic heart failure. Eur Heart J 27: 65-75.

43. Kwon BJ, Kim DB, Jang SW, Yoo KD, Moon KW, et al. (2010) Prognosis of heart failure patients with reduced and preserved ejection fraction and coexistent chronic obstructive pulmonary disease. Eur J Heart Fail 12: 1339-1344.

44. Linssen GC, Rienstra M, Jaarsma T, Voors AA, van Gelder IC, et al. (2011) Clinical and prognostic effects of atrial fibrillation in heart failure patients with reduced and preserved left ventricular ejection fraction. Eur J Heart Fail 13: 1111-1120. 